\title{
UNIDADES DE PESQUISAS PARA O DESENVOLVIMENTO SOCIAL: ANÁLISE DAS ATIVIDADES DO INSTITUTO BRASILEIRO DE INFORMAÇÃO EM CIÊNCIA E TECNOLOGIA, E DO OBSERVATÓRIO NACIONAL
}

\author{
CENTRO DE INVESTIGACIÓN PARA EL DESARROLLO \\ SOCIAL: ANÁLISIS DE LAS ACTIVIDADES DEL INSTITUTO \\ BRASILEÑO DE INFORMACIÓN EN CIENCIA Y \\ TECNOLOGÍA, Y DEL OBSERVATORIO NACIONAL
}

Diego Andres Salcedo - salcedo.da@gmail.com Doutor em Comunicação pela Universidade Federal de Pernambuco (UFPE). Professor do Departamento de Ciência da Informação da UFPE.

RESUMO

Introdução: Pressupõe que a relação entre a produção científica e a sociedade necessita de constante processo de retro-alimentação. Leva em conta o importante papel sócio-científico do Governo Federal brasileiro ao incentivar e avaliar, de forma constante, as ações e os financiamentos das Unidades de Pesquisa.

Objetivo: Propõe estabelecer uma relação entre as orientações do Ministério de Ciência, Tecnologia e Inovação voltadas ao desenvolvimento social e as atividades de duas Unidades de Pesquisa, a saber: Instituto Brasileiro de Informação em Ciência e Tecnologia e o Observatório Nacional.

Metodologia: Utiliza como procedimentos metodológicos a identificação, descrição e análise, tanto por meio de revisão bibliográfica, quanto dos conteúdos disponibilizados nas páginas eletrônicas dessas Unidades de Pesquisa.

Resultados: Ao considerar os parâmetros expressos na Ação Estratégica IV, intitulada "Ciência, Tecnologia e Inovação para o Desenvolvimento da Sociedade", foi observado que essa ação, em particular, visa disponibilizar para a sociedade, a 
partir das ações das Unidades de Pesquisa, projetos e programas voltados ao desenvolvimento social.

Conclusões: Verificou que, apesar de existir comunicação e certa concordância efetiva entre os órgãos envolvidos, algumas demandas sociais não são cumpridas, particularmente, no que diz respeito as metas de popularização e disseminação das informações científicas e tecnológicas.

Palavras-chaves: Desenvolvimento Social. Instituto Brasileiro de Informação em Ciência e Tecnologia. Ministério da Ciência, Tecnologia e Inovação. Observatório Nacional. Unidades de Pesquisa.

\section{INTRODUÇÃO}

De acordo com Silveira, Pinheiro e Bazzo (2010), as transformações tecnológicas causam impactos tão rápidos na sociedade, que dificultam o acompanhamento dos seus resultados no contexto social. A partir dessas mudanças tecnológicas, torna-se de grande relevância a reflexão acerca do impacto que as mesmas trazem para nossa sociedade. O uso do conhecimento científico e tecnológico como ferramenta de transformação social pode auxiliar nas relações entre sociedade, Estado e setor privado.

Para tanto partimos da premissa de que o acesso à informação em todos os contextos, principalmente nos relacionados a informações geradas pelo setor público, é assegurado por lei. Nesse sentido, instituições ligadas ao Governo Federal devem, de acordo com a Lei de Acesso à Informação Pública no 12.527 de Novembro de 2011, prover à sociedade acesso irrestrito as informações de interesse coletivo, que não possuam características sigilosas.

Com isso, este estudo verificou se as diretrizes apontadas pelo Ministério da Ciência, Tecnologia e Inovação (MCTI), voltadas para o desenvolvimento social, são seguidas pelas Unidades de Pesquisa (UP's), a partir de seus programas e projetos. Assim, as UP's analisadas foram o Instituto Brasileiro de Informação em Ciência e Tecnologia (IBICT), e o Observatório Nacional (ON).

Para à análise e compreensão do assunto em questão, foi realizada nos sites do MCTI, do IBICT e do ON, uma comparação entre as informações dispostas em relação as atividades que são realizadas nessas UP's e que estão diretamente 
ligadas as orientações do MCTI acerca da Ciência, Tecnologia e Inovação (CT\&l) para o Desenvolvimento Social no Brasil.

Por fim, a partir dessas comparações, é possível identificar quais as informações que, de fato, estão disponíveis para atender as demandas da sociedade no que tange à CT\&l, e se existe a preocupação em popularizar e disseminar essas informações à sociedade, seguindo o princípio legal da informação pública.

\section{DESENVOLVIMENTO SOCIAL}

Desde o período da industrialização, em meados do século XIX, algumas sociedades passam por profundas e rápidas transformações tecnológicas. Estas, por sua vez, constituem e contribuem para um discurso voltado, em grande medida, ao desenvolvimento econômico como condição ao crescimento do país. O capitalismo trouxe a cultura do progresso científico e tecnológico representando o caminho para o progresso humano, porém o objetivo continuava sendo a lucratividade, sem tanta preocupação com o desenvolvimento social.

A tecnologia, na maioria das vezes, é utilizada como sinônimo de desenvolvimento socioeconômico. Por outro lado, rememora Salcedo (2010, p. 21), "a estreita relação entre a Ciência e a Tecnologia é tema das agendas políticas e econômicas da grande maioria dos países. Relação tão complexa que acarretou o surgimento dos neologismos tecnociências ou sóciotecnocientíficas". Enfim, e não é à toa, os países que possuem tecnologias avançadas, aliadas ao seu desenvolvimento socioeconômico, são considerados e denominados desenvolvidos, o que, claramente, configura um discurso de exclusão, ou de distinção entre centro e periferia.

Porém, é importante refletir até que ponto as tecnologias levam ao desenvolvimento de uma sociedade, ou seja, se todas as camadas da população conseguem fazer uso dessas tecnologias para melhorarem suas condições de vida. É preciso uma avaliação crítica acerca dos produtos e serviços resultantes da ciência e tecnologia e seus impactos no contexto social.

Nesse sentido, Motoyama (2004, p. 17) acredita que por C\&T

serem engrenagens essenciais do processo de desenvolvimento econômico e social da atualidade, bem ou mal, elas habitam o nosso cotidiano sem, no entanto, tornar-se parte de nossa cultura mais

Inf. Inf., Londrina, v. 19, n. 1, p. 76 - 94, jan./abr. 2014. http:www.uel.br/revistas/informacao/ 
geral. Pior: no seio da população brasileira, corre solta a lenda de que as atividades de C\&T não são para nós, mas dos outros, dos estrangeiros, dos naturais do Hemisfério Norte, abençoados pelo pensamento científico e pela habilidade tecnológica.

Investimentos em ciência e tecnologia podem trazer benefícios à sociedade, diminuindo as desigualdades sociais e garantindo o bem-estar coletivo, a partir da sua aplicação em setores básicos como educação, saúde e habitação. Ainda, as vantagens tecnológicas provenientes dos conhecimentos científicos podem ser aplicadas nas mais diversas áreas, propiciando a melhoria das condições sociais.

O uso das tecnologias resulta em soluções efetivas de transformação social e para que isso se torne constante é preciso que haja articulação entre a comunidade científica, governos, empresas e sociedade, no sentido de criar e manter um ambiente produtivo e sustentável para todos.

Não obstante, Salcedo (2010, p. 23) defende o ponto de vista de que

identificar, descrever e analisar as semelhanças e dessemelhanças entre a ciência e a tecnologia, tanto com relação aos aspectos qualitativos, quanto aos quantitativos, envolve inúmeras variáveis partícipes de complexos processos históricos. Outrossim, a ciência e a tecnologia são socialmente essenciais e, também, apenas duas das variáveis que constituem a cultura humana.

As diretrizes que orientam as políticas para o desenvolvimento social precisam ser debatidas através de um sistema participativo, com o objetivo de definir como as demandas sociais de Ciência e Tecnologia (C\&T) do país podem ser atendidas, considerando não, apenas, as diferentes regiões, culturas, necessidades e especificidades, mas, também, "o conhecimento como um dos principais instrumentos de superação das desigualdades" (RESENDE, 2005, p.6).

Nesse sentido, Haddad (2005, p. 11) corrobora com a assertiva de Resende, ao dizer que:

é preciso fomentar e investir no desenvolvimento científico e tecnológico, também é indispensável democratizar e direcionar a ciência e a tecnologia para o atendimento das demandas locais, contribuindo para o enfrentamento das desigualdades regionais e sociais do país, o que demanda uma ação sistêmica na educação, com políticas de acesso e qualidade em todos os níveis e modalidades de ensino, da alfabetização à pós-graduação [...]. A 
educação é o alicerce para a inclusão social, geração de trabalho e renda e para o desenvolvimento científico e tecnológico do país.

Por sua vez, Silveira, Pinheiro e Bazzo (2010, p. 6) admitem que

numa sociedade em que o desenvolvimento científico e tecnológico tornou-se hegemônico, é fundamental refletir sobre a tecnologia numa outra perspectiva. O que temos visto é que o progresso tecnológico não tem atendido às necessidades básicas da população, mas servido para a promoção de interesses de poucos como estratégia do sistema capitalista.

Para este estudo, as demandas sociais serão consideradas como fatores determinantes de ações em C\&T para o desenvolvimento do país. A partir disso, o acesso a informação pública pode ser identificado, avaliado e analisado, se considerado como um objeto de estudo nuclear no campo da C\&T, como um dos principais assuntos que precisa ser discutido no sentido de verificar o que o governo está fazendo nesse âmbito.

A Lei 12.527, também conhecida como Lei de Acesso à Informação Pública, regulamenta o acesso à informação sob a guarda do Estado, a qual é um bem público, com o objetivo de consolidar a democracia, promover a cidadania e informar a sociedade acerca dos programas e projetos executados pelo governo. Isso possibilita que os cidadãos, quando bem informados, tenham autonomia na tomada de decisões sobre questões que lhes dizem respeito, podendo acessar informações, por exemplo, sobre o que se tem feito em relação aos direitos essenciais como saúde, educação etc.

A Lei de Acesso à Informação Pública também estabelece que as informações de interesse coletivo devem estar disponibilizadas na Internet para o acompanhamento de programas e projetos. Considerando essa prerrogativa legal, analisamos a página eletrônica do Ministério da Ciência, Tecnologia e Inovação (MCTI), para identificar quais são as propostas de programas voltadas para o desenvolvimento social. Além disso, verificar se as Unidades de Pesquisa (UP's), vinculadas ao $\mathrm{MCTI}$, escolhidas para este estudo, seguem ou estão de alguma forma articuladas com as diretrizes sugeridas pelo $\mathrm{MCTI}$, quais sejam: Observatório Nacional (ON), e Instituto Brasileiro de Informação em Ciência e Tecnologia (IBICT). 


\section{UNIDADES DE PESQUISAS (UP's)}

Para tanto, se faz importante saber que as UP's são instituições ligadas a Pesquisa e Desenvolvimento (P\&D) sustentadas por políticas do Governo Nacional, contando ainda com o incentivo de instituições de fomento e até mesmo com parcerias de empresas privadas, com a finalidade de apoiar as Universidades a promover, desenvolver e disseminar pesquisas.

Pesquisas essas que podem contribuir significativamente com a sociedade ao qual estão diretamente relacionadas. Além disso, de acordo com Cilone (2012), as UP's surgiram, com a intenção de alavancar o ranking da produção científica e de inovação do Brasil.

Desta forma, pode-se ainda entender por UP's, como sendo locais onde um indivíduo ou grupos deles que tenham interesses comuns dentro das áreas a que se destinam atuar, desenvolvendo pesquisas, que venham a ter como resultado, a produção de conhecimentos; produtos palpáveis provenientes desses conhecimentos e de conhecimentos já antes produzidos e agora aplicados, ou até mesmo, resultem em algum serviço.

Todas as pesquisas e resultados desenvolvidos numa UP têm caráter significativo da possibilidade de solucionar problemas ou atender necessidades e demandas provenientes da sociedade. Assim, elas podem contribuir para o desenvolvimento dos níveis sociais, auxiliando na intelectualização dos grupos, apontar possíveis melhorias para problemas sociais, e dar subsídios para o desenvolvimento econômico de determinadas localidades.

Para tanto, as próximas subseções apresentarão um breve histórico e definições relacionadas as UP's e suas atividades, as quais serão analisadas neste trabalho.

\subsection{Instituto Brasileiro de Informação em Ciência e Tecnologia (IBICT)}

O Instituto Brasileiro de Informação em Ciência e Tecnologia (IBICT) é uma Unidade de pesquisa (UP's), vinculada ao MCTI que promove o acesso a informações a respeito de Ciência, Tecnologia e Inovação (CT\&I).

Sobre a sua origem pode-se inferir que: 
Por meio de proposta conjunta CNPq/FGV [Conselho Nacional de Pesquisa/Fundação Getúlio Vargas], foi criado, em 27 de fevereiro de 1954, pelo Decreto do presidente da República $n^{\circ} 35.124$, o Instituto Brasileiro de Bibliografia e Documentação (IBBD), que passou a integrar a estrutura organizacional do CNPq. (INSTITUTO BRASILEIRO DE INFORMAÇÃO EM CIÊNCIA E TECNOLOGIA, 2012).

Em 1976 o IBBD se transforma no atual IBICT. Em 2000, o IBICT passa a ser subordinado ao Ministério da Ciência e Tecnologia (MCT), o qual se torna o MCTI. Sua sede fica localizada em Brasília e no Rio de Janeiro funciona seu núcleo de Ensino e Pesquisa, resultado de um convênio do IBICT com a Universidade Federal do Rio de Janeiro (UFRJ). Este ano, no mês de fevereiro, no Rio de Janeiro foram inauguradas novas instalações.

Estas instalações possibilitarão, de acordo com o diretor do IBICT, Emir José Suaiden (2013), que o Instituto ganhe mais visibilidade, proporcione um ambiente mais agradável com suporte tecnológico adequado e dê um maior suporte para os novos pesquisadores e para a demanda do Programa de Pós-Graduação em Ciência da Informação (PPGCI - IBICT/UFRJ).

Possui a missão de promover a competência, o desenvolvimento de recursos e a infraestrutura de informação em Ciência e Tecnologia para a produção, a socialização e a integração do conhecimento científico-tecnológico. Visando continuar se mantendo como centro de excelência em Ciência da Informação, além de alcançar as condições político institucionais, infraestruturais e de competência científico-tecnológica para atender às demandas e aos novos desafios em ciência, tecnologia e inovação, colocados pela sociedade do conhecimento.

Sabe-se que o IBICT é uma referência quando se fala em promoção de acesso a informação em CT\&I e para que sua missão e visão sejam alcançadas satisfatoriamente, ele atua realizando atividades como: transferir tecnologias de informação; editorar revistas; formar e capacitar recursos humanos para pesquisa na área de Ciência da Informação; desenvolver pesquisas, serviços e produtos de informação tecnológica; distribuir o conhecimento produzido nas suas áreas de atuação; realizar programas e projetos de cooperação e parcerias estabelecidas em acordos, convênios, de caráter nacional e internacional. 
Dentro das suas diversas atuações, podemos citar a Biblioteca Digital Brasileira de Teses e Dissertações (BDTD), que reúne as teses e dissertações dos profissionais das diferentes áreas do conhecimento do país, disponibilizando-as de forma integral e gratuita pela internet a todos os interessados. O Canal Ciência é outro projeto de incentivo à educação científica, como também um portal de divulgação, o qual deseja através de suas ações, aproximar a sociedade do mundo das ciências e, para isso, eles utilizam uma linguagem de fácil entendimento para o público em geral, expondo suas produções intelectuais em formato digital.

Temos ainda, a Revista de Inclusão Social que publica trabalhos visando incluir os cidadãos na sociedade da informação e do conhecimento, abordando assuntos como inclusão e acessibilidade digital, podendo ser obtida de forma impressa ou eletrônica. A Rede Brasileira de Serviços de Preservação Digital (Cariniana) é uma parceria do IBICT com a Stanford University para criação de serviços de preservação digital. Segundo o Coordenador da Cariniana, Miguel Angel Mardero Arellano (2012) "o objetivo principal desta rede é disponibilizar serviços de preservação digital de recursos exclusivamente eletrônicos". As diversas e diferentes atuações do IBICT possibilitam que o país cresça e se desenvolva de forma sólida e contínua com relação à produção, disseminação, forma de preservação e socialização das informações referentes ao âmbito da CT\&I.

\subsection{Observatório Nacional (ON)}

O Observatório Nacional (ON) é uma das mais antigas instituições brasileiras de pesquisa, ensino e prestação de serviços tecnológicos, foi o primeiro a atuar na área da Astronomia, da Geofísica e da Metrologia de tempo e frequência, estabelecendo as bases dessas áreas do conhecimento.

Segundo o Padre Serafim Leite (apud OBSERVATÓRIO NACIONAL, 2009), foi criado em 1730, quando os jesuítas instalaram um observatório no Morro do Castelo, no Rio de Janeiro. Em 1780 um observatório foi montado no mesmo local, pelos astrônomos portugueses Sanches d'Orta e Oliveira Barbosa, realizando-se ali observações regulares de astronomia, meteorologia e magnetismo terrestre. Mas, oficialmente foi fundado pelo Imperador D. Pedro I em 15 de outubro de 1827, instalado no torreão da Escola Militar. 
Desde 1920 está instalado no Morro de São Januário, em São Cristóvão, no Rio de Janeiro, preservando o seu patrimônio histórico e mantendo modernas instalações de pesquisas com equipamentos de última geração, que acompanham a evolução tecnológica de suas áreas, tornando-se Unidade de Pesquisa (UP's) do CNPq em 1976. Em 1999, o ON ficou subordinado ao Ministério da Ciência e Tecnologia (MCT), atualmente MCTI.

Sua atuação é baseada na cooperação científica entre instituições e grupos de pesquisa, em parcerias com empresas e disseminação do conhecimento para a sociedade. Para melhorar sua atuação, vem investindo na intensificação das colaborações internacionais, na diversificação das parcerias com empresas, no fortalecimento de seus cursos de pós-graduação e no aumento das ações de interlocução com a sociedade e a comunidade científica.

Inicialmente foi criado devido à necessidade de obter com maior exatidão, facilidade e segurança através dos instrumentos e profissionais experientes, a declinação magnética, a hora média e a longitude, para regulação dos cronômetros e realização de viagens marítimas seguras, além da necessidade de conhecer os elementos geográficos de pontos do território com o objetivo de construir mapas.

Atualmente tem a missão de realizar pesquisa e desenvolvimento em Astronomia, Geofísica e Metrologia em tempo e frequência, formar pesquisadores em seus cursos de pós-graduação, capacitar profissionais, coordenar projetos e atividades nestas áreas e gerar, manter e disseminar a Hora Legal Brasileira, visando ter reconhecimento nacional e projeção internacional com destacada atuação em suas áreas de competência.

Para que esse reconhecimento nacional e projeção internacional pudessem ser alcançados, o ON fez projetos estruturantes, que objetivaram chegar aos resultados previstos. Tais projetos estão expostos no plano diretor de unidade de 2011 a 2015, contendo quatro projetos estruturantes. O projeto estruturante IMPACTON, de acordo com a Coordenação de Astronomia e Astrofísica (2011), tem o objetivo de pesquisar pequenos corpos do Sistema Solar em órbitas próximas a terra, prevendo o risco de colisão destes com a mesma.

Para realizar essa pesquisa, o ON instalou e operacionalizou o Observatório Astronômico do Sertão de Itaparica (OASI), possibilitando dessa forma, que o ON e 
o país se integrassem aos programas internacionais de busca e seguimento de asteróides e cometas em risco de colisão com a Terra.

Através do OASI, localizado no município de Itacuruba em Pernambuco, é possível realizar observação de pequenos corpos celestes, através de seu telescópio robótico, produzir publicações científicas, e colaborar com outras instituições e projetos do $\mathrm{ON}$, integrando objetos de pesquisa e formando profissionais.

O projeto estruturante PAU-BRASIL realiza levantamento de dados astronômicos voltados para o estudo da rápida expansão do universo, para determinar principalmente a equação de estado da chamada energia escura, que é um dos problemas fundamentais da cosmologia atual, resultante da atual descoberta da acelerada expansão do universo.

Ainda, o projeto PAU-BRASIL, representa o ON e compila suas ações com relação a sua participação na colaboração internacional Brasil-Espanha para o desenvolvimento do Javalambre Physics of the Accelerating Universe Astrophysical Survey (J-PAS), que envolve o gerenciamento e construção das câmeras para dois telescópios robóticos no Pico del Buitre, em Teruel, Espanha. Devido à criação de uma nova técnica para utilização de multifiltros com largura fixa, será possível a produção de uma gigantesca quantidade de dados que interessarão a todas as áreas em que a astronomia Brasileira atua.

O projeto estruturante ASTROSOFT II fortalece suas ações criando um centro de dados astronômicos atuando como um laboratório nacional - Laboratório Interinstitucional de e-Astronomia (LIneA) - em parceria com o Centro Brasileiro de Pesquisas Físicas (CBPF) e o Laboratório Nacional de Computação Científica (LNCC), dando continuidade ao projeto ASTROSOFT. Este, por sua vez, foi criado para dar suporte à participação brasileira nos projetos Dark Energy Survey (DES), o qual tem o objetivo de estudar a natureza da energia escura, que representa mais de $50 \%$ do conteúdo do Universo e é a responsável pela aceleração de sua expansão e no Sloan Digital Sky Survey III (SDSS-III) que faz um levantamento de dados astronômicos da extensa área do céu.

Para levantamento de dados relacionados aos mapeamentos do céu, são criadas ferramentas para tratar, analisar, armazenar e tornar públicos os dados e produtos científicos desses mapeamentos. Tanto o acesso aos dados, quanto a 
utilização das ferramentas de análise são geridas por meio de um portal científico construído por pesquisadores e técnicos brasileiros, que possibilitaria o aumento da participação do Brasil nesses projetos.

O projeto estruturante Rede Brasileira de Observatórios e Padrões Geofísicos (REBOG) foi criado para dar suporte ao desenvolvimento cientifico e tecnológico da área. Tem como objetivo melhorar as atividades de coleta, processamento, gestão e disseminação dos dados procedentes dos monitoramentos de fenômenos geofísicos no Brasil. O REBOG, atualmente, é composto de quatro projetos, o Banco de Dados Ambientais para a Indústria do Petróleo (BAMPETRO), o Pool de Equipamentos Geofísicos do Brasil (PEGBr), a Rede Brasileira de Observatórios Magnéticos (REBOM) e a Rede Sismográfica do Sul e do Sudeste do Brasil(RSIS).

Vale ressaltar, que o PEGBr e o RSIS contam com recursos da Petrobrás, por intermédio de sua Rede Temática de Geotectônica, focando na capacidade instrumental e de geração de infraestrutura para pesquisa. Ainda, participa da Rede de Geofísica Aplicada da Petrobrás que custeia os estudos de métodos geofísicos, que se relacionem com as áreas do pré-sal na bacia de Santos.

Será constatado o sucesso desse projeto, quando forem analisados os indicadores de integração da rede com suas semelhantes nacionais e internacionais de apoio à pesquisas relacionadas com os diversos fenômenos geofísicos, e de subsídio às atividades sócio-econômicas desenvolvidas nas áreas de Geofísica, Geodésia e Metrologia.

Percebe-se, então, que o ON na área de geofísica almeja principalmente fortalecer o seu papel como laboratório nacional, por isso, presta serviços diversificados, produz pesquisas básicas e, em parceria como setor produtivo, participa de projetos de produção e desenvolvimento, atuando nós vários temas que a Geofísica abrange, focando em Geomagnetismo e Geoeletricidade, Gravimetria, Sismologia, Instrumentação Geofísica e Geotermia.

$\mathrm{Na}$ área de Metrologia de tempo e freqüência são realizados projetos de pesquisa voltados para a produção de algoritmos de sincronização com certificação, aperfeiçoamento da rastreabilidade nacional e internacional em tempo e freqüência e criação de métodos para aprimoramento da Melhor Capacidade de Medição. Esta área é responsável por guardar os padrões nacionais de tempo e frequência, que são a base da Rastreabilidade Metrológica Brasileira de Tempo e Frequência, 
utilizando sua capacidade técnica e instrumental para atender às necessidades de empresas e da sociedade em geral.

Para isso, criou a Rede de Sincronismo à Hora Legal Brasileira (ReSincHLB), a Rede de Carimbo de Tempo (ReTempHLB) e oferece serviços de calibração de equipamentos e de sincronismo público à Hora Legal Brasileira. São enfatizadas nas suas colaborações internacionais, a especialização de pessoal, a interação técnicocientífica e inserção em redes de rastreabilidade.

Percebemos que as ações concebidas, através de seus projetos, nas áreas de Astronomia, Geofísica e Metrologia de tempo e frequência, conforme o plano diretor do Observatório Nacional (2011-2015), possibilitam que o ON tenha um avanço intelectual nas suas áreas de atuação, colaborando para a melhoria interna da instituição, aumentando a sua capacidade na realização de pesquisa tanto de cooperação nacional como internacional, consolidando sua presença em outras regiões, estabelecendo parcerias com diferentes instituições para ver de que forma o projeto será realizado e como se manterá, ocasionando crescimento na produção científica e na formação de profissionais, intensificando a colaboração com as outras unidades de pesquisa do $\mathrm{MCTI}$, desenvolvendo produtos para a comunidade científica, contribuindo, assim, para o desenvolvimento do país.

\section{$4 \quad$ UNIDADES DE PESQUISAS PARA A DEMANDA SOCIAL}

Tendo em vista toda a necessidade social de avanço e mudança, o governo, por meio MCTI, passou a apontar para áreas que são de suma importância para o desenvolvimento social, buscando contribuir cada vez mais com essas ações. Para isso, foram criadas UP's, que abrigam pesquisadores e incentivam-nos a trabalhar em prol da ciência fora dos domínios das Universidades, mas em parceria com elas, dando a esses pesquisadores mais uma infraestrutura de qualidade e possibilidade de exploração dos seus campos de interesse, ou seja, as UP's surgiram de acordo com Cilone (2012), a partir da estratégia do governo federal com o intuito de criar um ambiente favorável para a inovação.

No entanto, vale ressaltar que a implantação dessas Unidades não se dá apenas com 0 intuito de acolher pesquisadores em locais para o seu próprio desenvolvimento profissional/acadêmico, mas antes de tudo, pensando como essa 
classe da sociedade pode vir a ter maior visibilidade e contribuição para com o desenvolvimento de uma região através seus estudos.

Cilone (2012), ainda, diz que o desenvolvimento das UP's veio com a finalidade maior de estabelecer padrões e estratégias de proteção e transferência dos resultados adquiridos nos laboratórios para a sociedade. Ou seja, as Unidades de Pesquisa, em sua essência, possuem um dever e caráter de contribuição para o desenvolvimento social, dando a região, as quais estão alocadas, algumas soluções para os seus eventuais problemas.

Além da implantação dessas UP's, o MCTI criou ainda linhas de ações que devem ser seguidas por essas unidades, buscando sempre o bem comum a todos de modo interno e externo a elas, tendo sempre como alvo o desenvolvimento social. De acordo com o site do Ministério, são identificadas quatro linhas de ações, as quais estão baseadas basicamente em (I) Expandir e Consolidar o Sistema Nacional de C,\&I; (ii) Promover Inovação Tecnológica em Empresas; (iii) Pesquisar, Desenvolver e Inovar em Áreas Estratégicas; e por fim, utilizar a (iv) Ciência, Tecnologia e Inovação para o Desenvolvimento da Sociedade. Desta forma, todas as UPs ligadas ao MCTI, devem seguir em seus planos de ações, essas quatro diretrizes, buscando compreender e atender às necessidades de cada região, ao qual estejam inseridas, independente das áreas que se destinam a atuar.

Como dito anteriormente, as UP's a serem estudadas e analisadas nesse trabalho, serão o Instituto Brasileiro de Informação em Ciência e Tecnologia (IBICT) e o Observatório Nacional (ON). Essas duas instituições possuem acima de tudo e qualquer um que sejam os seus objetivos, a função social de disseminação dos resultados de seus projetos e pesquisa, tendo em vista que todos os seus projetos são apoiados, influenciados e financiados pelo Governo Federal e instituições de fomento, que repassam parcelas dos impostos pagos pela sociedade como um todo. Desta forma, fazem-se necessários que todas as UP's, relacionem as suas atuações com os indicadores das quatro ações determinadas pelo MCTI. Para este trabalho, será avaliado a aplicação e desenvolvimento da ação quatro (Ciência, Tecnologia e Inovação para o Desenvolvimento da Sociedade), por essas duas UP's aqui apresentadas.

Buscou-se aqui, perceber se as ações propostas tanto pelo MCTI quanto pelas próprias instituições estão alinhadas e sendo cumpridas, além de observar 
quais as contribuições sociais alcançadas por essas instituições, e se a sociedade de modo geral consegue aperceber-se dos impactos causados pelas atividades desenvolvidas por essas unidades, ou se apenas uma parcela da sociedade é atendida por elas. Para isso, foi necessária a análise dos sites das instituições, focando o conteúdo disposto por elas, tentando chegar à compreensão das suas atividades e dos pontos que elas indicam o cumprimento da ação quatro do Ministério em suas atividades.

Em paralelo, foi feito ainda a análise do site do MCTI com o intuito de comparar a descrição das UP's analisadas e das atividades que elas devem cumprir, desta forma, tornou-se possível compreender o que são as Unidades em seus conceitos, o que elas deveriam ser em sua essência e o que elas de fato são, fazem e representam nas suas práticas do dia a dia, para a sociedade em geral. De acordo com MCTI, o IBICT é referência na promoção do acesso à informação sobre CT\&I. Seus programas criam interações dos resultados de suas pesquisas com as comunidades cientificas e tecnológicas, para colocar em prática o desenvolvimento de soluções voltadas ao bem estar social.

Entre as iniciativas está o Serviço Brasileiro de Respostas Técnicas (SBRT), que é gratuito e serve de apoio a empreendedores e micro e pequenas empresas ofertando soluções tecnológicas; o Programa de Avaliação do Ciclo de Vida (ACV), o qual foi desenvolvido para avaliar o impacto ambiental de bens e serviços; e Sistema Brasileiro de Informação em Tecnologia Industrial Básica (Info TIB), sistema de coleta,organização e integração de informações entre oferta e demanda de serviços tecnológicos no Brasil.

Tais iniciativas produzem resultados tão expressivos, que atraem instituições para acordo de cooperação voltado à informação científica, tecnológica e de inovação, além de criar ampla visão do potencial de produção para parcerias internacionais, as quais tenham interesse em conectar as pesquisas brasileiras.

Levando em consideração a sociedade como um todo e as ações para o desenvolvimento social, o IBICT possui o mapa de inclusão digital, que, por meio de metodologia própria, transfere tecnologias para apoiar o planejamento de ações locais de inclusão digital, assessorando e mantendo mapeados os pontos de inclusão digital em funcionamento no Brasil. 
Em parceria com a iniciativa privada, o IBICT desenvolve o inventário do ciclo de vida da competitividade ambiental da indústria brasileira, com objetivo de criar uma infraestrutura de informação capaz de suportar o armazenamento e a disseminação de informações de inventários da produção industrial brasileira.

O Observatório Nacional (ON) ainda busca o reconhecimento nacional na sociedade, onde criou projetos estruturantes descritos anteriormente. Apesar do contato com a sociedade científica através de projetos que produzem publicações científicas e integram seus programas para formações de profissionais através de especializações oferecidas, o resultado de suas pesquisas por ser um conteúdo de grande especificidade, ainda faz parte de um trabalho pouco disseminado pelo ambiente científico, sendo pouco explorado e conhecido pela sociedade em geral.

As ações concebidas, através de seus projetos, nas áreas de Astronomia, Geofísica e Metrologia de tempo e frequência, conforme o plano diretor do Observatório Nacional (2011-2015) possibilitam, que o ON tenha um avanço intelectual nas suas áreas de atuação, colaborando para a melhoria interna da instituição, aumentando a sua capacidade na realização de pesquisa tanto de cooperação nacional como internacional, consolidando sua presença em outras regiões, estabelecendo parcerias com diferentes instituições para ver de que forma o projeto será realizado e como se manterá, ocasionando crescimento na produção científica e na formação de profissionais, intensificando a colaboração com as outras unidades de pesquisa do $\mathrm{MCTI}$, desenvolvendo produtos para a comunidade científica, contribuindo, assim, para o desenvolvimento do país

Em caráter de atenção as orientações da ação IV do MCTI, o ON criou a divisão de atividades educacionais, com a missão de trazer o instituto para inclusão social. A Divisão de Atividades Educacionais (DAED) é responsável pela participação em feiras de C\&T, planejar a elaboração de projetos de publicação de revistas, folhetos, cartazes e outros meios de divulgação e de produção de materiais didáticos.

Entre as iniciativas estão as animações com conteúdo relacionado à astronomia, como pequeno cientista que incentiva a inovação e produção desde infância,estimulando o interesse pela temática desenvolvida no instituto e introduzindo esse contato com a base da educação (ensino fundamental e 
médio).Na mesma linha vem o brincando com a ciência ,que permite o contato com experiências básicas, trazendo o convívio com o conteúdo desde novo.

Ainda, na divisão de atividades educacionais, a ON criou a coleção de astronomia na escola, onde diversos livretos de linguagem simples, abordam temáticas desenvolvida pelo instituto. Na mesma linha está a revista em quadrinho com um especialista no assunto, pesquisador do observatório, transformando seu conhecimento crítico e rebuscado em linguagem simples e acessível para sociedade em geral.

As iniciativas acima identificadas, tanto do IBICT quanto do ON, estão de acordo com os propósitos do $\mathrm{MCTI}$, no que tange ao desenvolvimento social, colocando em comunicação todas as esferas sociais para produção e divulgação das informações científicas e tecnológicas, contribuindo, assim, para o bem estar social através do acesso à informação.

\section{CONSIDERAÇÕES FINAIS}

Tendo em vista tudo o que foi analisado, pode-se considerar que o Governo Federal, através do MCTI, criou políticas e Unidades que contribuem ou pelo menos tentem contribuir com o avanço social, apostando no atendimento das necessidades e especificidades de diversas regiões, abrangendo, assim, a sociedade de modo geral.

É fato que a sociedade vem, com o passar dos tempos, buscando cada vez mais, informações e conhecimentos, de modo que possam, construir seu pensamento crítico acerca de assuntos que envolvam as tomadas de decisões dos principais fatores de desenvolvimento socioeconômico do país. E nesse ponto, as Unidades de Pesquisas possuem em seus índices de desenvolvimento, indicados pelo Ministério nas suas quatro ações, responsabilidades em dar a esses indivíduos, informações necessárias para que se tornem cada vez mais capazes de racionalizar sobre suas vidas e seus ambientes.

Desta forma, a partir do que foi proposto neste trabalho, em relação a observação do cumprimento da ação IV do Ministério pelo IBICT e ON, tendo em vista que essa observação deu-se a partir das páginas web dessas duas instituições, sobre o que elas se propõem a fazer, em comparação ao que o MCTI indica que 
elas devam de fato fazer, pode-se observar que dentro do plano de ação, o qual está disposto nos sites, as Unidades estão em concordância com o Ministério, não deixando de cumprir com suas responsabilidades.

Vale ressaltar que, a análise foi desenvolvida apenas a partir de informações dispostas nas páginas dos sites em relação as atividades desenvolvidas pelas Unidades em seus laboratórios. O estudo aqui exposto não possui caráter exaustivo, ficando essas análises resguardadas para trabalhos futuros. Podendo buscar a fundo, se as pesquisas e os seus resultados, os quais estão sendo indicados pelas unidades como em andamento, estão de fato sendo aplicados na sociedade, e qual o peso dessa contribuição.

Por fim, este primeiro escrito indica a necessidade de continuidade dos trabalhos de pesquisa com vistas a explorar alguns elementos fundamentais de análise como: produção científica; impacto dos serviços prestados; análise de custobenefício de pesquisa etc., aspectos relevantes para aprimorar o debate sobre o papel social atrelado às pesquisas científicas realizadas pelas UP's no Brasil. Tudo isso pode servir para que um pensar estratégico seja interposto junto aos planejamentos já existentes, particularmente, no que diz respeito aos novos modos de produção, circulação e disseminação do conhecimento científico e tecnológico.

\section{REFERÊNCIAS}

BRASIL. Lei. $\mathrm{n}^{\circ} 12.527$, de 18 de novembro de 2011. Dispões sobre o acesso à Informação Pública. Diário Oficial da República Federativa do Brasil, Poder Executivo, Brasília, DF, 18 nov., 2011, Edição Extra. Disponível em: <http://www.planalto.gov.br/ccivil_03/_ato2011-2014/2011/lei/12527.htm>.

CILONE, Arquimedes D. Políticas e Ações do MCTI para apoio aos Núcleos de Inovação Tecnológicas - NITS. Disponível em <http://www.openinnovationseminar. com.br/2012/apresentacoes/arenas/institutos/ArquimedesDiogenes_MCTI.pdf>.

HADDAD, Fernando. Educação para induzir e democratizar o conhecimento científico. Inclusão Social, Brasília, v. 1, n. 1, p. 10-11, out./mar., 2005.

INSTITUITO BRASILEIRO DE INFORMAÇÃO EM CIÊNCIA E TECNOLOGIA. Disponível em: <http://www.ibict.br>.

MINISTÉRIO DE CIÊNCIA, TECNOLOGIA E INOVAÇÃO. Unidades de Pesquisas. Disponível em: <http://www.mcti.gov.br/>. 
MOTOYAMA, Shozo. Prelúdio para uma História: ciência e tecnologia no Brasil. São Paulo: EDUSP, 2004.

OBSERVATÓRIO NACIONAL. Disponível em: <http://www.on.br>.

RESENDE, Sérgio. Conhecimento e inclusão social. Inclusão Social, Brasília, v. 1, n. 1, p. 6-7, out./mar., 2005.

\section{SALCEDO, Diego A. A ciência nos selos postais comemorativos brasileiros:} 1900-2000. Recife: EDUFPE, 2010.

SILVEIRA, R. M. C. F., PINHEIRO, N. A. M., BAZZO, W. A. A Perspectiva Social do Desenvolvimento Científico e Tecnológico. Revista de Ensino de Engenharia, Passo Fundo, v. 29, n. 1, p. 3-10, 2010. Disponível em: <http://www.upf.br/seer/index .php/ree/article/view/262>.

\section{Title}

Research units for social development: activities analysis of the Brazilian Institute of Information in Science and Technology, and the National Observatory

\section{Abstract}

Introduction: Assumes that the relationship between scientific production and society requires constant process of feed-back. Takes into account the important socio-scientific role played by the Brazilian Federal Government to encourage and evaluate steadily, actions and funding of its research units.

Objective: Proposes to establish a relationship between the guidelines of the Ministry of Science, Technology and Innovation focused on social development and the activities of two of its research units, namely: Brazilian Institute of Information in Science and Technology and National Observatory.

Methodology: Methodological procedures were the identification, description and analysis both through literature review, as the content available from the websites of these research units.

Results: Considering the parameters expressed in the Strategic Action IV entitled "Science, Technology and Innovation for the Development of Society", it was observed that this action, in particular, aims to provide to society, from the actions of the Research Units, projects and programs for social development.

Conclusions: As a conclusion it was verified that, although there is effective communication and certain accordance between the agencies involved, some social demands are not fulfilled, particularly as regards to the goals of popularization and dissemination of scientific and technological information.

Key words: Desarrollo Social. Instituto Brasileño de Información en Ciencia y Tecnología. Ministerio de la Ciencia, Tecnología y Innovación. Observatorio Nacional. Unidades de Investigación. 


\section{Título}

Unidades de pesquisas para o desenvolvimento social: análise das atividades do Instituto Brasileiro de Informação em Ciência e Tecnologia, e do Observatório Nacional

\section{Resumen}

Introducción: Se supone que la relación entre la comunidad científica y la sociedad requiere un constante proceso de retro-alimentación. Toma en cuenta el importante rol socio-científico del Gobierno Federal de Brasil en fomentar y evaluar de manera constante las acciones y financiación de las unidades de investigación.

Objectivo: Propone establecer una relación entre las directrices del Ministerio de Ciencia, Tecnología e Innovación centrada en el desarrollo social y las actividades de dos unidades de investigación, a saber: Instituto Brasileño de Información en Ciencia y Tecnología y el Observatorio Nacional.

Metodología: Fueron utilizados como procedimientos metodológicos la identificación, descripción y análisis, tanto a través de revisión de la literatura, como el contenido disponible en los sitios web de estas unidades de investigación.

Resultados: Al considerar los parâmetros expresados en la Acción Estratégica IV intitulada "Ciencia, Tecnología y Innovación para el Desarrollo de la Sociedad", se observó que esa acción, en particular, tiene como objetivo proporcionar a la sociedad, a partir de las acciones de las unidades de investigación, proyectos y programas orientadas al desarrollo social.

Conclusións: Encontró que, aunque existe una comunicación efectiva y cierto acuerdo entre las agencias involucradas, algunas de las demandas sociales no se cumplen, en particular en lo que respecta a los objetivos de divulgación y difusión de la información científica y tecnológica.

Palabras clave: Brazilian Institute of Information in Science and Technology. Ministry of Science, Technology and Innovation. National Observatory. Research Units. Social Development.

Recebido em: 12.10 .2013

Aceito em: 03.02.2014 